\title{
Hipoglicemiantes orales para el tratamiento de la diabetes mellitus gestacional. Revisión sistemática de la literatura
}

\author{
Milenko Pavlovic B. ${ }^{1}$, Jorge Carvajal C., PhD. ${ }^{2}$ \\ ${ }^{1}$ Programa de Obstetricia y Ginecología, ${ }^{2}$ Unidad de Medicina Materno Fetal. División de Obstetricia y Ginecología, \\ Facultad de Medicina, Pontificia Universidad Católica de Chile.
}

\section{RESUMEN}

Antecedentes: La diabetes mellitus gestacional (DMG) se asocia a mayor riesgo materno y perinatal. El manejo habitual de ésta patología es la dieta, el ejercicio y la insulina. Los hipoglicemiantes orales (HGO) son una terapia emergente para el tratamiento de la DMG. Objetivos: Realizar una revisión sistemática de toda la evidencia tipo I disponible acerca del uso de HGO para tratamiento de DMG y realizar un metaanálisis de los resultados maternos y perinatales significativos. Resultados: Diez estudios cumplieron criterios de selección. Tres estudios comparaban metformina vs insulina, cuatro gliburide vs insulina y tres metformina vs gliburide. Los estudios no encontraron diferencias significativas en control glicémico ni en complicaciones perinatales entre metformina vs insulina, gliburide vs insulina y metformina vs gliburide. Nuestro metaanálisis mostró que la glicemia de ayuno es significativamente menor (DM 1,74; IC95\% 0,383,10 ) y la glicemia postprandial a las 2 horas es significativamente mayor en el grupo insulina vs HGO (DM $-2,97 ;$ IC95\% - 27,24 a -5,36). Nuestro metaanálisis muestra que la incidencia de fetos grandes para edad gestacional fue significativamente menor en el grupo metformina vs gliburide (OR 0,38; IC95\% 0,18-0,78). El fracaso del tratamiento con gliburide fue significativamente menor que con metformina $(27,6 \%$ vs $38,5 \%$, p<0,0001; IC95\% 1,21-1,60). Conclusión: Los HGO son un tratamiento seguro y efectivo para DMG. Recomendamos gliburide (glibenclamida) para el tratamiento de las pacientes con DMG que fracasan su control glicémico con dieta y ejercicio, por no cruzar la placenta, tener menor tasa de fallo y ser igualmente efectiva que metformina.

\section{PALABRAS CLAVE: Diabetes mellitus gestacional, hipoglicemiantes orales, metformina, gliburide, glibenclamida, insulina}

\section{SUMMARY}

Background: Gestational diabetes mellitus (GDM) is associated to a higher maternal and perinatal risk. Usually GDM is controlled with diet, exercise and insulin. Oral hypoglycaemic agents (OHA) are an emergent therapy for the treatment of GDM. Objectives: Conduct a systematic review of all class I evidence available regarding the use of OHA for GDM treatment, and perform a metaanalysis of significant maternal and perinatal outcomes. Results: Ten studies accomplished inclusion criteria. Three studies compared metformin to insulin, four compared glyburide to insulin and three compared metformin to glyburide. Studies showed no significant differences in glycaemic control or perinatal complications, between metformin and insulin, between glyburide and insulin, or between metformin and glyburide. Our metaanalysis comparing OHA to insulin shows significantly lower fasting blood glucose (MD 1.74; 95\% IC 0.38-3.10) and larger 2-hr postprandial glucose in the insulin group compared to OHA groups (MD -2.97; 95\% IC -27.24-5.36). Our metaanalysis comparing shows a significantly lower incidence of large for gestational age in the metformin vs. gliburide group (OR $0.38 ; 95 \%$ IC $0.18-0.78$ ). Failure of treatment was significantly lower using gliburide than metformin (27.6\% vs. $38.5 \%, p<0.0001 ; 95 \%$ IC 1.21-1.60). Conclusion: OHA are a safe and effective 
treatment for GDM. We recommend the use of glyburide (glibenclamide) in GDM patients that fail to obtain glycemic control with diet and exercise, since glyburide does not crosses the placental barrier, has a lower rate of treatment failure and is equally affective as metformin.

\section{KEY WORDS: Gestational diabetes mellitus, oral hypoglycemic agents, metformin, glyburide, glibenclamide, insulin}

\section{INTRODUCCIÓN}

Diabetes mellitus gestacional (DMG) se define como cualquier intolerancia a los hidratos de carbono que comienza o es diagnosticada por primera vez durante el embarazo (1). La incidencia de la DMG varía entre las distintas razas y según la metodología que se utilice para su diagnóstico. Se ha calculado la incidencia de DMG entre un 2,2\% y $15 \%$ (2).

La DMG se asocia a un mayor riesgo materno y perinatal. Los riesgos maternos incluyen desarrollo de preeclampsia durante el embarazo, mayor tasa de parto por cesárea y desarrollo de diabetes mellitus tipo 2 (DM2) a futuro. Los riesgos perinatales que aumentan con la DMG son: macrosomía fetal (MCSF), fetos grandes para la edad gestacional (GEG), distocia de hombros, lesiones fetales al momento del parto, hipoglicemia neonatal, síndrome de distrés respiratorio (SDR) e hiperbilirrubinemia (1).

El manejo clínico de la DMG reduce la incidencia de MCSF y de morbilidad perinatal. Para el tratamiento inicial de la DMG, se utiliza la dieta (restricción de hidratos de carbono) y el ejercicio. Cuando la dieta y el ejercicio no logran un adecuado control glicémico, es necesaria la utilización de insulina $(3,4)$.

La insulina tiene ventajas y desventajas para el tratamiento de la DMG. Entre las ventajas se encuentra que la insulina no cruza la barrera placentaria y es altamente efectiva para el control glicémico materno. Las desventajas del tratamiento de la DMG con insulina son que la insulina es una terapia cara, de difícil manejo, requiere de entrenamiento para su uso, debe guardarse bajo condiciones especiales, su uso se asocia a hipoglicemia y aumento de peso materno; todas estas dificultades pueden producir mala adherencia al tratamiento y ser de difícil implementación en el escenario de bajos recursos $(5,6)$.

Los hipoglicemiantes orales (HGO) son una alternativa emergente a nivel mundial para el tratamiento de la DMG, cuando la dieta y el ejercicio fallan en su manejo. Los HGO tienen ventajas y desventajas para el tratamiento de la DMG. Las ventajas son su bajo costo, fácil administración y amplia disponibilidad. Entre las desventajas se encuentran que estos cruzan la barrera placentaria, podrían producir hipoglicemia neonatal, podrían producir teratogenicidad y no están aprobados por la US Food and Drug Administration (FDA) para tra- tamiento de la DMG $(7,8)$.

Dos familias de HGO han sido utilizadas en el manejo de la DMG: las sulfonilureas de segunda generación (gliburide y glibenclamida) y las biguanidas (metformina). Las sulfonilureas de segunda generación, aumentan la secreción de insulina por las células pancreáticas y no cruzan la barrera placentaria $(9,10)$. Las biguanidas inhiben la gluconeogénesis hepática y aumentan la sensibilidad de los receptores del músculo y tejido graso a la glucosa $(11,12)$. La metformina cruza la barrera placentaria en forma significativa, pero existe evidencia que muestra que no tiene efectos adversos fetales, neonatales, ni a 18 meses de vida $(13,14)$.

El objetivo de este trabajo es realizar una revisión sistemática de toda la evidencia tipo I (estudios clínicos randomizados controlados) disponible acerca del uso de HGO en el tratamiento de DMG y realizar un metaanálisis de los resultados maternos y perinatales significativos.

\section{MATERIALES Y MÉTODOS}

Se realizó una búsqueda en la base de datos de MEDLINE utilizando la siguiente estrategia: (Therapy/Broad[filter]) AND ("Diabetes, Gestational"[Mesh] OR "GDM") AND ("Metformin"[Mesh] OR "biguanides" OR "Glyburide"[Mesh] OR "sulfonylurea" OR "HypoglycemicAgents"[Mesh]). Los criterios de inclusión fueron:

1. El diseño de los trabajos debe ser estudios randomizados controlados.

2. La población a estudiar debe ser mujeres cursando embarazos únicos con diagnóstico de DMG. 3. Las intervenciones a comparar deben ser HGO vs insulina o HGO vs HGO.

4. El idioma del trabajo debe ser inglés o español.

Se realizó un metanálisis de los resultados maternos y perinatales susceptibles a metaanalizar, utilizando el software Review Manager 5 de la Cochrane Collaboration. Se utilizó la prueba estadística de Mantel-Haenszel para variables dicotómicas y la prueba estadística de Varianza Inversa para variables continuas. Para variables dicotómicas se calculó la razón de Odds con intervalo de confianza de $95 \%$ (IC95\%) y para las variables continuas se calculó la diferencia de medias (DM) con IC95\%.

Se realizó la comparación entre las tasas globales de fracaso de metformina y gliburide, utilizando la prueba de Chi cuadrado. 


\section{RESULTADOS}

Se encontraron 434 trabajos con nuestra estrategia de búsqueda, los cuales se filtraron por "clinical trials", obteniéndose luego de esto 76 publicaciones. De estas 76 publicaciones, sólo 10 artículos cumplieron los criterios de inclusión.

De los 10 artículos que cumplían los criterios de inclusión, 3 comparaban metformina vs insulina $(5,11,15), 3$ comparaban gliburide vs insulina
$(9,16,17), 3$ comparaban gliburide vs metformina $(6,10,12)$ y uno comparaba gliburide vs acarbosa vs insulina (18). Pudimos acceder al texto completo de 9 artículos, del artículo 15 sólo pudimos acceder al resumen. Para nuestro análisis, obtuvimos los datos crudos de este trabajo (15) de un metaanálisis (2) que incluía sus resultados. Las características de los estudios y los resultados de interés son presentados en las Tablas I-V.

Tabla I

CARACTERÍSTICAS DE DISEÑO DE LOS ESTUDIOS INCLUIDOS

\begin{tabular}{|c|c|c|c|c|c|c|}
\hline Autor & Grupos & $\begin{array}{l}\text { Criterios } \\
\text { Selección }\end{array}$ & $\begin{array}{l}\text { Resultado } \\
\text { Primario }\end{array}$ & $\begin{array}{l}\text { Cálculo } \\
\text { Tamaño } \\
\text { Muestral }\end{array}$ & $\begin{array}{l}\text { Análisis } \\
\text { Intención } \\
\text { de Tratar }\end{array}$ & $\begin{array}{c}\text { Pérdida } \\
\text { Seguimiento } \\
\text { (no analiza) }\end{array}$ \\
\hline \multicolumn{7}{|l|}{ Metformina vs Insulina } \\
\hline $\begin{array}{l}\text { Rowan et al. 2008, } \\
\text { Australia y } \\
\text { N. Zelandia (5) }\end{array}$ & $\begin{array}{l}\text { M: } 373 \\
\mathrm{I}: 378\end{array}$ & $\begin{array}{l}\text { Inclusión: Si } \\
\text { Exclusión: Si }\end{array}$ & $\begin{array}{l}\text { Compuesto de } \\
\text { complicaciones } \\
\text { neonatales }\end{array}$ & $\mathrm{Si}$ & $\mathrm{Si}$ & $\begin{array}{l}\text { M: } 10(2,68 \%) \\
\text { I: } 8(2,11 \%)\end{array}$ \\
\hline $\begin{array}{l}\text { ljäs et al. 2010, } \\
\text { Finlandia (11) }\end{array}$ & $\begin{array}{l}\mathrm{M}: 47 \\
\mathrm{I}: 50\end{array}$ & $\begin{array}{l}\text { Inclusión: Si } \\
\text { Exclusión: Si }\end{array}$ & MCSF y GEG & $\mathrm{Si}$ & $\mathrm{Si}$ & Sin pérdida \\
\hline $\begin{array}{l}\text { Moore et al. 2007, } \\
\text { USA (15) }\end{array}$ & $\begin{array}{l}\mathrm{M}: 32 \\
\mathrm{I}: 31\end{array}$ & $\begin{array}{l}\text { Inclusión: Si } \\
\text { Exclusión: Si }\end{array}$ & Control glicémico & NE & NE & Sin pérdida \\
\hline \multicolumn{7}{|l|}{ Gliburide vs Insulina } \\
\hline $\begin{array}{l}\text { Langer et al. } 2000 \text {, } \\
\text { USA (9) }\end{array}$ & $\begin{array}{l}\text { G: } 201 \\
\text { I: } 203\end{array}$ & $\begin{array}{l}\text { Inclusión: Si } \\
\text { Exclusión: No }\end{array}$ & Control glicémico & No & Si & Sin pérdida \\
\hline $\begin{array}{l}\text { Anjalakshi et al. 2007, } \\
\text { India (17) }\end{array}$ & $\begin{array}{l}\text { G: } 13 \\
\mathrm{I}: 13\end{array}$ & $\begin{array}{l}\text { Inclusión: Si } \\
\text { Exclusión: No }\end{array}$ & Control glicémico & No & NE & $\begin{array}{l}\text { G: } 3(23 \%) \\
\text { I: } 0\end{array}$ \\
\hline $\begin{array}{l}\text { Ogunyemi et al. 2007, } \\
\text { USA (16) }\end{array}$ & $\begin{array}{l}\text { G: } 48 \\
\text { I: } 49\end{array}$ & $\begin{array}{l}\text { Inclusión: No } \\
\text { Exclusión: No }\end{array}$ & $\begin{array}{l}\text { Control glicémico } \\
\text { y peso del RN }\end{array}$ & No & $\mathrm{Si}$ & No especifica \\
\hline $\begin{array}{l}\text { Bertini et al. 2005, } \\
\text { Brasil (18) }\end{array}$ & $\begin{array}{l}\text { G: } 24 \\
\text { A: } 19 \\
\text { I: } 27\end{array}$ & $\begin{array}{l}\text { Inclusión: Si } \\
\text { Exclusión: Si }\end{array}$ & $\begin{array}{l}\text { Hipoglicemia del } \\
\text { RN y peso RN }\end{array}$ & No & NE & Sin pérdida \\
\hline \multicolumn{7}{|l|}{ Metformina vs Gliburide } \\
\hline $\begin{array}{l}\text { Moore et al. 2010, } \\
\text { USA (12) }\end{array}$ & $\begin{array}{l}\text { M: } 75 \\
\text { G: } 74\end{array}$ & $\begin{array}{l}\text { Inclusión: Si } \\
\text { Exclusión: Si }\end{array}$ & Control glicémico & No & $\mathrm{Si}$ & Sin pérdida \\
\hline $\begin{array}{l}\text { Silva et al. 2010, } \\
\text { Brasil (6) }\end{array}$ & $\begin{array}{l}\text { M: } 32 \\
\text { G: } 40\end{array}$ & $\begin{array}{l}\text { Inclusión: Si } \\
\text { Exclusión: Si }\end{array}$ & $\begin{array}{l}\text { Control glicémico, } \\
\text { peso RN y Glice- } \\
\text { mia neonatal }\end{array}$ & No & NE & Sin pérdida \\
\hline $\begin{array}{l}\text { Silva et al. 2012, } \\
\text { Brasil (10) }\end{array}$ & $\begin{array}{l}\text { M: } 104 \\
\text { G: } 96\end{array}$ & $\begin{array}{l}\text { Inclusión: Si } \\
\text { Exclusión: Si }\end{array}$ & $\begin{array}{l}\text { Control glicémico, } \\
\text { peso RN y Glice- } \\
\text { mia neonatal }\end{array}$ & No & NE & Sin pérdida \\
\hline
\end{tabular}

A: Acarbosa , M: Metformina, G: Gliburide, I: Insulina, GEG: Grande para edad gestacional, MCSF: Macrosomía fetal, NE: No especifica, RN: Recién nacido. 
Tabla II

CARACTERÍSTICAS GENERALES DE LOS ESTUDIOS INCLUIDOS

\begin{tabular}{|c|c|c|c|}
\hline Autor & $\begin{array}{l}\text { Criterios Dg de } \\
\text { DMG (PTGO) }\end{array}$ & $\begin{array}{l}\text { Objetivos Control } \\
\text { Glicémico }\end{array}$ & $\begin{array}{c}\text { Dosis HGO } \\
\text { Inicio / Máxima } \\
\text { (mg / día) }\end{array}$ \\
\hline \multicolumn{4}{|l|}{ Metformina vs Insulina } \\
\hline $\begin{array}{l}\text { Rowan et al. 2008, } \\
\text { Australia y } \\
\text { N. Zelandia (5) }\end{array}$ & $\begin{array}{l}\text { PTGO } 75 \mathrm{~g} \\
\text { A: } 99 \mathrm{mg} / \mathrm{dl} \\
\text { 2-h: } 144 \mathrm{mg} / \mathrm{dl}\end{array}$ & $\begin{array}{l}\text { A: } 97,2 \text { mg/dl } \\
\text { PP 2-h: } 120,6 \text { mg/dl }\end{array}$ & $500 / 2500$ \\
\hline $\begin{array}{l}\text { ljäs et al. 2010, } \\
\text { Finlandia (11) }\end{array}$ & $\begin{array}{l}\text { PTGO } 75 \mathrm{~g} \\
\text { A: } 95 \mathrm{mg} / \mathrm{dl} \\
\text { 2-h: } 173 \mathrm{mg} / \mathrm{dl}\end{array}$ & $\begin{array}{l}\text { A: } 95 \text { mg/dl } \\
\text { PP 2-h: } 120 \text { mg/dl }\end{array}$ & 750 / 2200 \\
\hline $\begin{array}{l}\text { Moore et al. } 2007 \text {, } \\
\text { USA (15) }\end{array}$ & $\begin{array}{l}\text { PTGO } 100 \mathrm{~g} \\
\text { A: } 105 \mathrm{mg} / \mathrm{dl} \\
\text { 3-h: } 145 \mathrm{mg} / \mathrm{dl}\end{array}$ & $\begin{array}{l}\text { A: } 105 \text { mg/dl } \\
\text { PP 2-h: } 120 \text { mg/dl }\end{array}$ & NE \\
\hline \multicolumn{4}{|l|}{ Gliburide vs Insulina } \\
\hline $\begin{array}{l}\text { Langer et al. } 2000 \text {, } \\
\text { USA (9) }\end{array}$ & $\begin{array}{l}\text { PTGO } 100 \mathrm{~g} \\
\text { A: } 95 \mathrm{mg} / \mathrm{dl} \\
\text { 3-h: NE }\end{array}$ & $\begin{array}{l}\text { A: } 95 \mathrm{mg} / \mathrm{dl} \\
\text { PP 2-h: } 120 \mathrm{mg} / \mathrm{dl}\end{array}$ & $2,5 / 20$ \\
\hline $\begin{array}{l}\text { Anjalakshi et al. } 2007 \text {, } \\
\text { India (17) }\end{array}$ & $\begin{array}{l}\text { PTGO } 75 \mathrm{~g} \\
\text { A: NE } \\
\text { 2-h: } 140 \mathrm{mg} / \mathrm{dl}\end{array}$ & $\begin{array}{l}\text { A: NE } \\
\text { PP 2-h: } 120 \text { mg/dl }\end{array}$ & 0,625 / NE \\
\hline $\begin{array}{l}\text { Ogunyemi et al. } 2007 \text {, } \\
\text { USA (16) }\end{array}$ & NE & NE & NE \\
\hline $\begin{array}{l}\text { Bertini et al. 2005, } \\
\text { Brasil (18) }\end{array}$ & $\begin{array}{l}\text { PTGO } 75 \mathrm{~g} \\
\text { A: } 110 \mathrm{mg} / \mathrm{dl} \\
\text { 2-h: } 140 \mathrm{mg} / \mathrm{dl}\end{array}$ & $\begin{array}{l}\text { A: } 90 \text { mg/dl } \\
\text { PP 2-h: } 100 \text { mg/dl }\end{array}$ & $\begin{array}{l}\text { G: } 5 / 20 \\
\text { Ac: } 50 \text { / } 300\end{array}$ \\
\hline \multicolumn{4}{|l|}{ Metformina vs Gliburide } \\
\hline $\begin{array}{l}\text { Moore et al. 2010, } \\
\text { USA (12) }\end{array}$ & $\begin{array}{l}\text { PTGO } 100 \mathrm{~g} \\
\text { A: } 105 \mathrm{mg} / \mathrm{dl} \\
\text { 3-h: } 145 \mathrm{mg} / \mathrm{dl}\end{array}$ & $\begin{array}{l}\text { A: } 105 \text { mg/dl } \\
\text { PP 2-h: } 120 \text { mg/dl }\end{array}$ & $\begin{array}{l}\text { M: } 500 / 2000 \\
\text { G: } 2,5 / 20\end{array}$ \\
\hline $\begin{array}{l}\text { Silva et al. 2010, } \\
\text { Brasil (6) }\end{array}$ & NE & $\begin{array}{l}\text { A: } 90 \text { mg/dl } \\
\text { PP 2-h: } 120 \text { mg/dl }\end{array}$ & $\begin{array}{l}\text { M: } 1000 / 2500 \\
\text { G: } 5 / 20\end{array}$ \\
\hline $\begin{array}{l}\text { Silva et al. 2012, } \\
\text { Brasil (10) }\end{array}$ & $\begin{array}{l}\text { PTGO } 75 \mathrm{~g} \\
\text { A: } 105 \mathrm{mg} / \mathrm{dl} \\
\text { 2-h: } 120 \mathrm{mg} / \mathrm{dl}\end{array}$ & $\begin{array}{l}\text { A: } 90 \mathrm{mg} / \mathrm{dl} \\
\text { PP 2-h: } 120 \mathrm{mg} / \mathrm{dl}\end{array}$ & $\begin{array}{l}\text { M: } 1000 \text { / } 2500 \\
\text { G: } 5 \text { / } 20\end{array}$ \\
\hline
\end{tabular}

2-h: Dos horas, 3-h: Tres horas, A: Ayuno, Ac: Acarbosa, Dg: Diagnóstico, DMG: Diabetes mellitus gestacional, G: Gliburide, M: Metformina, NE: No especificado, PP 2-h: Postprandial 2 horas, PTGO: Prueba tolerancia glucosa oral.

Metformina vs Insulina. Tres estudios compararon metformina vs insulina para el tratamiento de DMG con falla del manejo con dieta y ejercicio $(5,11,15)$. El estudio randomizado más grande que evalúa metformina para el tratamiento de la DMG, randomiza a 733 mujeres (5). Su resultado primario, un compuesto de complicaciones neonatales (hipoglicemia neonatal, SDR, necesidad de fotote- rapia, trauma en el parto, APGAR a los 5 minutos $<7$ y parto prematuro), no mostró diferencias significativas entre los grupos estudiados $(32,0 \%$ en el grupo metformina y $32,2 \%$ en el grupo insulina, $p=0,95)$. En el desglose del resultado primario, se aprecian algunas diferencias significativas: mayor frecuencia de hipoglicemia neonatal severa en el grupo insulina (insulina $8,1 \%$ vs metformina $3,3 \%$, 
Tabla III

RESULTADOS MATERNOS

\begin{tabular}{|c|c|c|c|c|c|c|}
\hline Autor & $\begin{array}{l}\text { Control } \\
\text { Glicémico }\end{array}$ & Hipoglicemia & $\begin{array}{l}\text { Aumento } \\
\text { Peso }\end{array}$ & PE & $\begin{array}{l}\text { Fallo } \\
\text { Tratamiento }\end{array}$ & $\begin{array}{l}\text { Tasa } \\
\text { Cesárea }\end{array}$ \\
\hline \multicolumn{7}{|l|}{ Metformina vs Insulina } \\
\hline $\begin{array}{l}\text { Rowan et al. } 2008 \\
\text { Australia y N. Zelandia (5) }\end{array}$ & $\begin{array}{l}\text { Met menor } \\
\text { glicemia PP } \\
\text { 2-h }(P=0,003)\end{array}$ & NE & $\begin{array}{l}\text { Menor Met } \\
(P=0,001)\end{array}$ & S/D & $46,3 \%$ & S/D \\
\hline $\begin{array}{l}\text { ljäs et al. 2010, } \\
\text { Finlandia (11) }\end{array}$ & NE & NE & S/D & S/D & $31,9 \%$ & $\begin{array}{l}\text { Mayor } \\
\text { Met } \\
(P=0,04)\end{array}$ \\
\hline $\begin{array}{l}\text { Moore et al. 2007, } \\
\text { USA (15) }\end{array}$ & S/D & NE & $\mathrm{NE}$ & $N E$ & $\mathrm{NE}$ & S/D \\
\hline \multicolumn{7}{|l|}{ Gliburide vs Insulina } \\
\hline $\begin{array}{l}\text { Langer et al. 2000, } \\
\text { USA (9) }\end{array}$ & S/D & $\begin{array}{l}\text { Menor Gli } \\
(P=0,003)\end{array}$ & NE & S/D & $4 \%$ & S/D \\
\hline $\begin{array}{l}\text { Anjalakshi et al. 2007, } \\
\text { India (17) }\end{array}$ & S/D & S/D & $\mathrm{NE}$ & $N E$ & $0 \%$ & NE \\
\hline $\begin{array}{l}\text { Ogunyemi et al. 2007, } \\
\text { USA (16) }\end{array}$ & $\begin{array}{l}\text { Gli mayor } \\
\text { glicemia PP 2-h } \\
(P=0,02)\end{array}$ & $\mathrm{S} / \mathrm{D}$ & $\mathrm{NE}$ & $N E$ & $7 \%$ & S/D \\
\hline $\begin{array}{l}\text { Bertini et al. 2005, } \\
\text { Brasil (18) }\end{array}$ & S/D & S/D & S/D & $N E$ & $\begin{array}{l}\text { G: } 20,8 \% \\
\text { Ac: } 42,1 \%\end{array}$ & S/D \\
\hline \multicolumn{7}{|l|}{ Metformina vs Gliburide } \\
\hline $\begin{array}{l}\text { Moore et al. 2010, } \\
\text { USA (12) }\end{array}$ & S/D & S/D & $\mathrm{NE}$ & S/D & $\begin{array}{l}\text { M: } 34,7 \% \\
\text { G: } 16,2 \%\end{array}$ & $\begin{array}{l}\text { Mayor } \\
\text { Met } \\
(P=0,02)\end{array}$ \\
\hline $\begin{array}{l}\text { Silva et al. 2010, } \\
\text { Brasil (6) }\end{array}$ & S/D & NE & $\begin{array}{l}\text { Menor Met } \\
(P=0,02)\end{array}$ & $N E$ & $\begin{array}{l}\text { M: } 25 \% \\
\text { G: } 23,8 \%\end{array}$ & S/D \\
\hline $\begin{array}{l}\text { Silva et al. 2012, } \\
\text { Brasil (10) }\end{array}$ & S/D & NE & $\begin{array}{l}\text { Menor Met } \\
(P=0,04)\end{array}$ & $N E$ & $\begin{array}{l}\text { M: } 21 \% \\
\text { G: } 29 \%\end{array}$ & S/D \\
\hline
\end{tabular}

Ac: Acarbosa, G: Gliburide, Gli: Gliburide, M: Metformina, Met: Metformina, NE: No especificado, PP 2-h: Postprandial 2 horas, PE: Preeclampsia, S/D: Sin diferencia.

$\mathrm{p}=0,008)$ y menor frecuencia de parto prematuro espontáneo en el grupo insulina (insulina $7,6 \%$ vs metformina $12,1 \%, p=0,04$ ); obviamente el estudio no tiene poder para hacer este análisis, ni el de los resultados secundarios que estudia (Tablas I-V).

El segundo estudio randomiza a 97 mujeres (11); su resultado primario fue la incidencia de MCSF o la incidencia de GEG, ambos analizados por separado. El estudio fue diseñado para mostrar una diferencia de un $30 \%$ en la incidencia de MCSF entre los dos grupos; sin embargo, para mostrar equivalencia entre ambos grupos el poder es insuficiente (se requeriría un tamaño muestral de 375 pacientes por cada grupo). En este estudio, el resultado primario no mostró diferencias significativas (MCSF: grupo insulina $22 \%$ vs grupo metformina $19,1 \%, p=0,72$; GEG: grupo insulina $10 \%$ vs grupo metformina 8,5\%, $\mathrm{p}=0,80$ ) (Tablas I-V).

El tercer estudio randomiza a 63 mujeres (15). Su resultado primario fue el control glicémico materno. Es un estudio pequeño, de metodología discutible (sin cálculo de tamaño muestral) que no mostró diferencias significativas ni para glicemia de ayuno ni para glicemia postprandial a las 2 horas (Tablas I-V). 


\section{Tabla IV}

RESULTADOS NEONATALES I

\begin{tabular}{|c|c|c|c|c|c|}
\hline Autor & MCSF & Peso RN & Hipoglicemia & SDR & $\begin{array}{l}\text { Admisión } \\
\text { UCl }\end{array}$ \\
\hline \multicolumn{6}{|l|}{ Metformina vs Insulina } \\
\hline $\begin{array}{l}\text { Rowan et al. 2008, } \\
\text { Australia y N. Zelandia (5) }\end{array}$ & $\mathrm{NE}$ & $S / D$ & $S / D$ & $S / D$ & $S / D$ \\
\hline $\begin{array}{l}\text { ljäs et al. 2010, } \\
\text { Finlandia (11) }\end{array}$ & $S / D$ & $S / D$ & $S / D$ & NE & $S / D$ \\
\hline $\begin{array}{l}\text { Moore et al. 2007, } \\
\text { USA (15) }\end{array}$ & $S / D$ & $S / D$ & $S / D$ & NE & NE \\
\hline \multicolumn{6}{|l|}{ Gliburide vs Insulina } \\
\hline $\begin{array}{l}\text { Langer et al. } 2000, \\
\text { USA (9) }\end{array}$ & $S / D$ & S/D & $S / D$ & $S / D$ & $S / D$ \\
\hline $\begin{array}{l}\text { Anjalakshi et al. 2007, } \\
\text { India (17) }\end{array}$ & $N E$ & $S / D$ & NE & NE & NE \\
\hline $\begin{array}{l}\text { Ogunyemi et al. 2007, } \\
\text { USA (16) }\end{array}$ & NE & S/D & $S / D$ & $N E$ & NE \\
\hline $\begin{array}{l}\text { Bertini et al. 2005, } \\
\text { Brasil (18) }\end{array}$ & $\mathrm{NE}$ & $S / D$ & $\begin{array}{l}\text { Mayor Gli } \\
(P=0,006)\end{array}$ & NE & NE \\
\hline \multicolumn{6}{|l|}{ Metformina vs Gliburide } \\
\hline $\begin{array}{l}\text { Moore et al. 2010, } \\
\text { USA (12) }\end{array}$ & $S / D$ & $\begin{array}{l}\text { Menor Met } \\
(P=0,02)\end{array}$ & $S / D$ & NE & $S / D$ \\
\hline $\begin{array}{l}\text { Silva et al. 2010, } \\
\text { Brasil (6) }\end{array}$ & $S / D$ & S/D & $S / D$ & NE & NE \\
\hline $\begin{array}{l}\text { Silva et al. 2012, } \\
\text { Brasil (10) }\end{array}$ & $\mathrm{NE}$ & $\begin{array}{c}\text { Menor Met } \\
(P=0,01)\end{array}$ & $S / D$ & NE & $S / D$ \\
\hline
\end{tabular}

Gli: Gliburide, Met: Metformina, MCSF: Macrosomía fetal, NE: No especificado, RN: Recién nacido, SDR: Síndrome de distrés respiratorio, S/D: Sin diferencia, UCl: Unidad de cuidados intensivos.

Gliburide vs Insulina. Cuatro estudios compararon gliburide vs insulina para el tratamiento de DMG con falla del manejo con dieta y ejercicio $(9,16-18)$. El primero es el estudio randomizado de mayor tamaño que compara gliburide con insulina y randomiza 404 mujeres (9). Su resultado primario es el control glicémico de las pacientes. El diseño de este estudio es deficiente: sin cálculo de tamaño muestral, distintos criterios de selección de pacientes para el mismo grupo de estudio y sin análisis de resultados según esta diferencia. El resultado primario no mostró diferencias entre los grupos estudiados tanto para glicemias de ayuno $(p=0,17)$, preprandiales $(p=0,17)$, postprandiales $(p=0,60)$ y promedios $(p=0,99)$. Tampoco hubo diferencia en la hemoglobina glicosilada $(p=0,12)($ Tablas I-V).

El segundo y el tercer estudio $(16,17)$, están publicados como cartas al editor en sus respectivas revistas y tienen metodologías bastante pobres, con errores de diseño. El estudio (17), randomiza 23 mujeres y tiene como resultado primario el control glicémico materno, el cual no muestra diferencias significativas entre los grupos de estudio. El estudio (16), randomiza 97 mujeres y tiene como resultado primario el control glicémico materno y el peso del recién nacido (RN), ambos analizados por separado. Muestra diferencias significativas sólo en la glicemia postprandial de 2 horas, siendo mayor 
Tabla V

RESULTADOS NEONATALES II

\begin{tabular}{|c|c|c|c|c|}
\hline Autor & Muerte Neonatal & Malformaciones & Parto Prematuro & Trauma Parto \\
\hline \multicolumn{5}{|l|}{ Metformina vs Insulina } \\
\hline $\begin{array}{l}\text { Rowan et al. 2008, } \\
\text { Australia y N. Zelandia (5) }\end{array}$ & NE & NE & $\begin{array}{l}\text { Mayor Met } \\
(P=0,04)\end{array}$ & $S / D$ \\
\hline $\begin{array}{l}\text { ljäs et al. 2010, } \\
\text { Finlandia (11) }\end{array}$ & $\mathrm{NE}$ & $\mathrm{NE}$ & NE & $\mathrm{NE}$ \\
\hline $\begin{array}{l}\text { Moore et al. 2007, } \\
\text { USA (15) }\end{array}$ & $\mathrm{NE}$ & $\mathrm{NE}$ & NE & $\mathrm{NE}$ \\
\hline \multicolumn{5}{|l|}{ Gliburide vs Insulina } \\
\hline $\begin{array}{l}\text { Langer et al. } 2000 \text {, } \\
\text { USA (9) }\end{array}$ & S/D & S/D & NE & NE \\
\hline $\begin{array}{l}\text { Anjalakshi et al. 2007, } \\
\text { India (17) }\end{array}$ & $\mathrm{NE}$ & $\mathrm{NE}$ & $\mathrm{NE}$ & $\mathrm{NE}$ \\
\hline $\begin{array}{l}\text { Ogunyemi et al. 2007, } \\
\text { USA (16) }\end{array}$ & $\mathrm{NE}$ & S/D & NE & NE \\
\hline $\begin{array}{l}\text { Bertini et al. 2005, } \\
\text { Brasil (18) }\end{array}$ & $\mathrm{NE}$ & NE & NE & $\mathrm{NE}$ \\
\hline \multicolumn{5}{|l|}{ Metformina vs Gliburide } \\
\hline $\begin{array}{l}\text { Moore et al. 2010, } \\
\text { USA (12) }\end{array}$ & $\mathrm{NE}$ & $\mathrm{NE}$ & NE & $S / D$ \\
\hline $\begin{array}{l}\text { Silva et al. 2010, } \\
\text { Brasil (6) }\end{array}$ & $\mathrm{NE}$ & $\mathrm{NE}$ & $\mathrm{NE}$ & $\mathrm{NE}$ \\
\hline $\begin{array}{l}\text { Silva et al. 2012, } \\
\text { Brasil (10) }\end{array}$ & S/D & $\mathrm{NE}$ & NE & $\mathrm{NE}$ \\
\hline
\end{tabular}

Met: Metformina, NE: No especificado, S/D: Sin diferencia.

en el grupo gliburide $(p=0,02)$. Por el bajo número de pacientes incluidas, estos dos estudios, no cuentan con el poder suficiente para encontrar diferencias en los resultados primarios ni secundarios que estudiaron (Tablas I-V).

El cuarto estudio (18), compara gliburide vs insulina vs acarbosa. Randomiza 70 mujeres y sus resultados primarios fueron hipoglicemia fetal y peso del RN al nacer, analizados por separado. El diseño de este estudio es deficiente, no presenta cálculo de tamaño muestral ni informa el poder utilizado; el bajo número de mujeres en cada grupo permite observar que no cuenta con el poder suficiente para encontrar diferencias en los resultados primarios ni secundarios que estudiaron. Sus resultados muestran diferencia significativa para hipoglicemia neonatal, siendo más frecuente en el grupo gliburide que en los otros dos (gliburide $33,3 \%$, ascarbosa $5,3 \%$ e insulina $3,7 \% ; p=0,006$ ).

Metformina vs Gliburide. Tres estudios comparan metformina vs gliburide para el tratamiento de DMG con falla del manejo con dieta y ejercicio $(6,10,12)$. El primero, randomiza a 149 mujeres y su resultado primario fue el control glicémico materno (12). El diseño de este estudio es deficiente, no presenta cálculo de tamaño muestral y no explicita si las mujeres incluidas en el estudio cursaban embarazos únicos o no. El resultado primario no mostró diferencias significativas tanto para glicemia de ayuno $(p=0,23)$ como para glicemia postprandial a las 2 horas $(p=0,28)$. 
El segundo y tercer estudio $(6,10)$, parecen contar con la misma cohorte de pacientes enroladas, ya que el grupo de investigación es el mismo, el trabajo se lleva a cabo en el mismo centro clínico y el diseño de los estudios es exactamente igual; ambos con metodología deficiente pues no presentan cálculo de tamaño muestral ni el poder estimado. Los dos estudios se diferencian solamente en el número de pacientes enroladas y en la duración del reclutamiento, siendo el estudio publicado en 2012 (10), un año más largo.

El estudio (6), randomiza 72 mujeres y sus resultados primarios fueron el control glicémico materno, peso del RN al nacer y glicemia neonatal, analizados por separado. Estos resultados primarios no mostraron diferencias significativas entre los grupos de estudio.

El estudio (10), randomiza 200 mujeres y sus resultados primarios fueron el control glicémico materno, peso del RN al nacer y glicemia neonatal, analizados por separado. De los resultados primarios estudiados, sólo se encontró diferencia significativa en el peso del RN, siendo menor en el grupo metformina $(p=0,01)$.

\section{Fracaso de manejo con HGO}

El fracaso de los $\mathrm{HGO}$ en el tratamiento de la DMG, ocurre cuando con dosis máxima de HGO no se logra el control glicémico adecuado de la paciente; la gran mayoría de los trabajos analizados publican la tasa de fracaso del uso de los HGO. La tasa de fracaso de metformina en el control glicémico de pacientes con DMG se encuentra entre $21 \%$ y $46,3 \%$, mientras que la tasa de fracaso de gliburide en el control glicémico de pacientes con DMG se encuentra entre $4 \%$ y $29 \%$.

Realizamos la comparación de la tasa de fracaso global de metformina vs la tasa de fracaso global de gliburide, encontramos que gliburide fracasa sig- nificativamente menos en el control glicémico que metformina $(27,6 \%$ vs $38,5 \%, p<0,0001$; IC95\% 1,21-1,60).

\section{Metaanálisis}

Realizamos un metaanálisis comparando HGO vs insulina o metformina vs gliburide. Las variables que se compararon en ambos metaanálisis fueron: GEG, hipoglicemia neonatal, glicemia de ayuno y glicemia postprandial a las 2 horas. Se eligieron estas variables pues sólo de ellas había datos suficientes. Las Figuras 1-8 grafican los resultados del metaanálisis para todas las variables estudiadas.

HGO vs Insulina. Los metaanálisis para GEG y para hipoglicemia neonatal, no mostraron diferencias significativas entre los grupos estudiados (GEG; OR 0,96; IC95\% 0,71-1,30), (hipoglicemia neonatal; OR 1,03; IC95\% 0,76-1,39) (Figuras 1, 2). El metaanálisis para glicemia de ayuno, muestra diferencias significativas entre los grupos estudiados, siendo la glicemia de ayuno significativamente menor en el grupo insulina (DM 1,74; IC95\% 0,38-3,10) (Figura 3). El metaanálisis para glicemia postprandial a las 2 horas, muestra diferencias significativas entre los grupos estudiados, siendo la glicemia postprandial a las 2 horas significativamente menor en el grupo HGO (DM -2,97; IC95\% -27,24 a -5,36) (Figura 4).

Metformina vs Gliburide. El metaanálisis para GEG, muestra diferencias significativas entre los grupos estudiados, siendo la prevalencia de GEG significativamente menor en el grupo metformina (OR 0,38; IC95\% 0,18-0,78) (Figura 5). Los metaanálisis para hipoglicemia neonatal, glicemia de ayuno y glicemia postprandial a las 2 horas, no mostraron diferencias significativas entre los grupos estudiados (hipoglicemia neonatal; OR 0,86; IC95\% 0,44-1,69), (glicemia ayuno; DM 0,02; IC95\% -2,32 a 2,35), (glicemia postprandial 2 horas; DM -0,91; IC95\% -4,63 a 2,81) (Figuras 6-8).

\begin{tabular}{|c|c|c|c|c|c|c|c|}
\hline Estudios & \multirow{2}{*}{$\begin{array}{r}\text { HGO } \\
\text { Eventos } \\
70\end{array}$} & \multirow{2}{*}{$\begin{array}{r}\text { Total } \\
363\end{array}$} & \multicolumn{2}{|c|}{$\begin{array}{c}\text { Insulina } \\
\text { Eventos Total }\end{array}$} & \multirow{2}{*}{$\begin{array}{l}\text { Peso } \\
62,8 \%\end{array}$} & \multirow{2}{*}{$\begin{array}{c}\begin{array}{c}\text { Odds Ratio } \\
\text { M-H, Fixed, 95\% IC }\end{array} \\
1,04[0,72-1,51]\end{array}$} & $\begin{array}{c}\text { Odds Ratio } \\
\text { M-H, Fixed, } 95 \% \text { IC }\end{array}$ \\
\hline Rowan 2008 & & & 69 & 370 & & & \\
\hline ljäs 2010 & 4 & 47 & 5 & 50 & $5,0 \%$ & $0,84[0,21-3,33]$ & \\
\hline Moore 2007 & 3 & 32 & 6 & 31 & $6,3 \%$ & $0,43[0,10-1,90]$ & \\
\hline Langer 2000 & 24 & 201 & 26 & 203 & $25,9 \%$ & $0,92[0,51-1,67]$ & \\
\hline Total $(95 \%$ IC) & & 643 & & 654 & $100,0 \%$ & $0,96[0,71-1,30]$ & \\
\hline Total eventos & 101 & & 106 & & & & \\
\hline $\begin{array}{l}\text { Heterogenicidad: } \\
\text { Test para efecto }\end{array}$ & $\begin{array}{l}1,36 ; \mathrm{df} \\
Z=0,25\end{array}$ & $\begin{array}{l}=3(P \\
(P=C\end{array}$ & $\begin{array}{l}=0,71) \\
0,80)\end{array}$ & $1^{2}=0 \%$ & & & 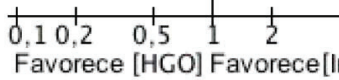 \\
\hline
\end{tabular}

Figura 1. Efectos de HGO vs insulina en GEG. 


\begin{tabular}{|c|c|c|c|c|c|c|c|c|}
\hline Estudios & $\begin{array}{r}\text { HGO } \\
\text { Eventos } \\
\end{array}$ & & \multicolumn{2}{|c|}{$\begin{array}{c}\text { Insulina } \\
\text { Eventos Total }\end{array}$} & \multirow{2}{*}{$\begin{array}{l}\text { Peso } \\
70,2 \%\end{array}$} & \multirow{2}{*}{$\begin{array}{c}\begin{array}{c}\text { Odds Ratio } \\
\text { M-H, Fixed, 95\% IC }\end{array} \\
0,78[0,53-1,15]\end{array}$} & \multicolumn{2}{|c|}{$\begin{array}{c}\text { Odds Ratio } \\
\text { M-H, Fixed, } 95 \% \text { IC }\end{array}$} \\
\hline Rowan 2008 & 55 & 363 & 69 & 370 & & & & \\
\hline ljäs 2010 & 4 & 47 & 7 & 50 & $7,5 \%$ & $0,57[0,16-2,09]$ & & - \\
\hline Moore 2007 & 0 & 32 & 2 & 31 & $3,0 \%$ & $0,18[0,01-3,94]$ & & \\
\hline Langer 2000 & 18 & 201 & 12 & 203 & $13,2 \%$ & $1,57[0,73-3,34]$ & & -- \\
\hline Ogunyemi 2007 & 12 & 48 & 6 & 49 & $5,4 \%$ & $2,39[0,82-7,00]$ & & \\
\hline Bertini 2005 & 8 & 24 & 1 & 27 & $0,8 \%$ & $13,00[1,48-113,87]$ & & \\
\hline Total $(95 \%$ IC) & & 715 & & 730 & $100,0 \%$ & $1,03[0,76-1,39]$ & & \\
\hline Total eventos & 97 & & 97 & & & & & \\
\hline $\begin{array}{l}\text { Heterogenicidad: } \mathrm{C} \\
\text { Test para efecto } \mathrm{g}\end{array}$ & $\begin{array}{l}12,76 ; d f \\
: Z=0,18\end{array}$ & $\begin{array}{l}f=5( \\
(P=\end{array}$ & $\begin{array}{l}=0,03) \\
, 86)\end{array}$ & $J^{2}-6$ & & & $\begin{array}{lc}0,01 & 0,1 \\
\text { Favorece }[\mathrm{HGO}]\end{array}$ & 1010100 \\
\hline
\end{tabular}

Figura 2. Efectos de HGO vs insulina en hipoglicemia neonatal

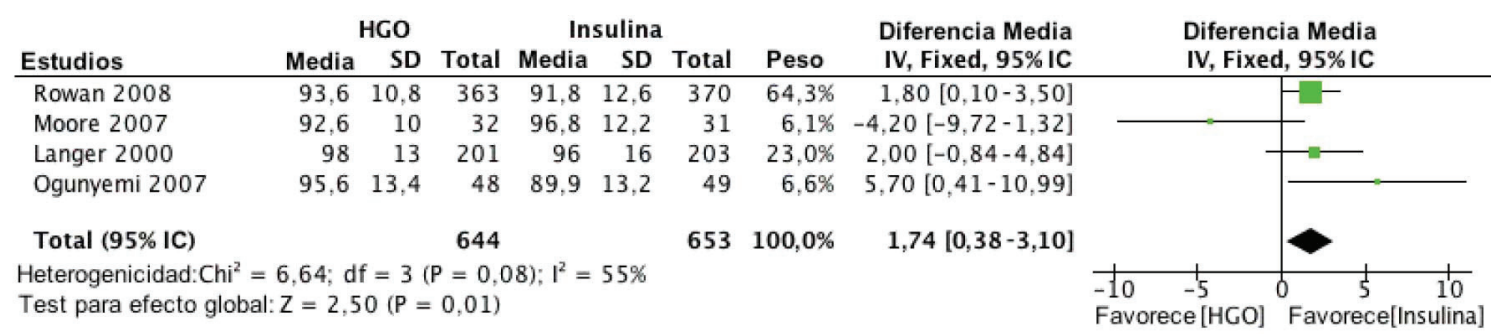

Figura 3. Efectos de HGO vs insulina en glicemia de ayuno.

\begin{tabular}{|c|c|c|c|c|c|c|c|c|c|c|}
\hline \multirow[b]{2}{*}{ Estudios } & \multicolumn{3}{|c|}{ HGO } & \multicolumn{3}{|c|}{ Insulina } & \multirow[b]{2}{*}{ Peso } & \multirow{2}{*}{$\begin{array}{r}\text { Diferencia Media } \\
\text { IV, Fixed, 95\% IC }\end{array}$} & \multirow{2}{*}{\multicolumn{2}{|c|}{$\begin{array}{l}\text { Diferencia Media } \\
\text { IV, Fixed, } 95 \% \text { IC }\end{array}$}} \\
\hline & Media & SD & Total & Media & SD & Total & & & & \\
\hline Rowan 2008 & 111,6 & 10,8 & 363 & 115,2 & 16,2 & 370 & $74,0 \%$ & $-3,60[-5,59--1,61]$ & & \\
\hline Moore 2007 & 106 & 13,9 & 32 & 112,5 & 32,9 & 31 & $1,9 \%$ & $-6,50[-19,04-6,04]$ & & \\
\hline Langer 2000 & 113 & 22 & 201 & 112 & 15 & 203 & $21,7 \%$ & $1,00[-2,68-4,68]$ & & - \\
\hline Ogunyemi 2007 & 116,3 & 16,6 & 49 & 132,6 & 35 & 48 & $2,4 \%$ & $-16,30[-27,24--5,36]$ & & \\
\hline Total $(95 \%$ IC) & & & 645 & & & 652 & $100,0 \%$ & $-2,97[-4,68--1,26]$ & $\bullet$ & \\
\hline $\begin{array}{l}\text { Heterogenicidad: } \mathrm{Cr} \\
\text { Test para efecto } \mathrm{gl}\end{array}$ & $\begin{array}{l}0,88 \\
z=3,4\end{array}$ & $\mathrm{df}=3$ & $P=0$, & $01) ; 1$ & $2 \%$ & & & & $\begin{array}{cc}-20 & -10 \\
\text { Favorece } & {[\mathrm{HGO}]}\end{array}$ & $\begin{array}{lcc}0 & 10 & 20 \\
\text { Favorece } & \text { [Insulina] }\end{array}$ \\
\hline
\end{tabular}

Figura 4. Efectos de HGO vs insulina en glicemia postprandial a las 2 horas

\begin{tabular}{|c|c|c|c|c|c|c|c|c|}
\hline \multirow{2}{*}{$\frac{\text { Estudios }}{\text { Silva } 2010}$} & \multicolumn{2}{|c|}{$\begin{array}{l}\text { Metformina } \\
\text { Eventos Total }\end{array}$} & \multicolumn{2}{|c|}{$\begin{array}{c}\text { Gliburide } \\
\text { Eventos Total }\end{array}$} & \multirow{2}{*}{$\frac{\text { Peso }}{28,7 \%}$} & \multirow{2}{*}{$\begin{array}{c}\begin{array}{c}\text { Odds Ratio } \\
\text { M-H, Fixed, 95\% IC }\end{array} \\
0,36[0,09-1,45]\end{array}$} & \multicolumn{2}{|c|}{$\begin{array}{c}\text { Odds Ratio } \\
\text { M-H, Fixed, } 95 \% \text { IC }\end{array}$} \\
\hline & 3 & 32 & 9 & 40 & & & & - \\
\hline Silva 2012 & 9 & 104 & 19 & 96 & $71,3 \%$ & $0,38[0,16-0,90]$ & & \\
\hline Total (95\% IC) & & 136 & & 136 & $100,0 \%$ & $0,38[0,18-0,78]$ & & \\
\hline Total eventos & 12 & & 28 & & & & & \\
\hline $\begin{array}{l}\text { Heterogenicidad: } \\
\text { Test para efecto }\end{array}$ & $\begin{array}{l}0,01 ; d f= \\
Z=2,64\end{array}$ & $\begin{array}{l}1(P= \\
(P=0,\end{array}$ & $\begin{array}{l}=0,93) ; \mathrm{I} \\
, 008)\end{array}$ & $0 \%$ & & & $\begin{array}{cc}0,01 & 0,1 \\
\text { Favorece [Metformina] }\end{array}$ & $\begin{array}{cc}10 & 100 \\
\text { Favorece[Gliburide] }\end{array}$ \\
\hline
\end{tabular}

Figura 5. Efectos de metformina vs gliburide en GEG. 


\begin{tabular}{|c|c|c|c|c|c|c|c|c|}
\hline \multirow{2}{*}{$\frac{\text { Estudios }}{\text { Moore } 2010}$} & Metformina & $\begin{array}{l}\text { nina } \\
\text { Total }\end{array}$ & \multicolumn{2}{|c|}{$\begin{array}{c}\text { Gliburide } \\
\text { Eventos Total }\end{array}$} & \multirow{2}{*}{$\frac{\text { Peso }}{5,5 \%}$} & \multirow{2}{*}{$\begin{array}{c}\text { Odds Ratio } \\
\text { M-H, Fixed, 95\% IC } \\
0,99[0,06-16,07]\end{array}$} & \multicolumn{2}{|c|}{$\begin{array}{c}\text { Odds Ratio } \\
\text { M-H, Fixed, 95\% IC }\end{array}$} \\
\hline & 1 & 75 & 1 & 74 & & & & \\
\hline Silva 2010 & 6 & 32 & 7 & 40 & $27,9 \%$ & $1,09[0,33-3,63]$ & & \\
\hline Silva 2012 & 11 & 104 & 13 & 96 & $66,7 \%$ & $0,76[0,32-1,78]$ & & \\
\hline Total $(95 \%$ IC) & & 211 & & 210 & $100,0 \%$ & $0,86[0,44-1,69]$ & & \\
\hline Total eventos & 18 & & 21 & & & & & \\
\hline $\begin{array}{l}\text { Heterogenicidad: } \\
\text { Test para efecto }\end{array}$ & $\begin{array}{l}0,24 ; \mathrm{df}= \\
Z=0,44\end{array}$ & $\begin{array}{l}=2(P= \\
(P=0\end{array}$ & $\begin{array}{l}=0,89): 1 \\
(66)\end{array}$ & $=0 \%$ & & & 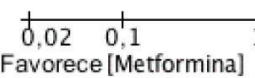 & $\begin{array}{cc}10 & 50 \\
\text { Favorece [Gliburide] }\end{array}$ \\
\hline
\end{tabular}

Figura 6. Efectos de metformina vs gliburide en hipoglicemia neonatal.

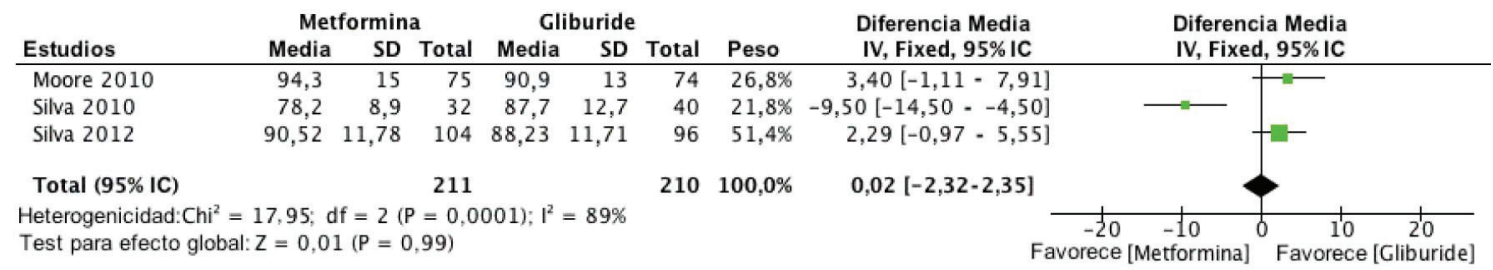

Figura 7. Efectos de metformina vs gliburide en glicemia de ayuno.

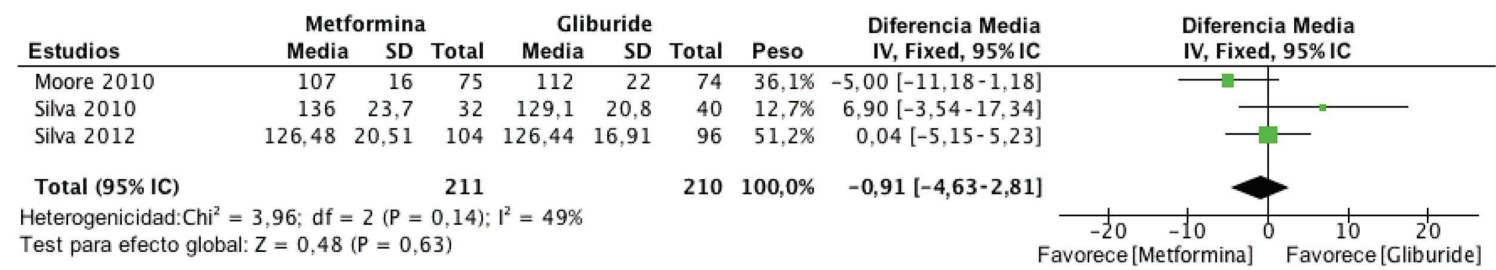

Figura 8. Efectos de metformina vs gliburide en glicemia postprandial a las 2 horas.

\section{DISCUSIÓN}

La DMG es una enfermedad frecuente en el embarazo, cuyo manejo está enfocado en evitar la hiperglicemia materna, causante de los efectos adversos maternos y perinatales de esta patología $(1,3,4)$. La terapia médica más utilizada en la DMG, cuando fracasa el manejo con dieta y ejercicio, es la insulina, la cual ha demostrado ser eficiente en el control glicémico y segura para el feto $(5,6)$. El uso de HGO para el manejo de la DMG es discutido, dado la escasa información acerca de la seguridad de su uso en relación al feto.

Nuestro trabajo analiza la mejor evidencia disponible acerca del uso de HGO para el manejo de la DMG. Se revisaron 10 estudios clínicos randomizados controlados, de los cuales 3 comparaban metformina vs insulina, 4 comparaban gliburide vs insulina y 3 comparaban metformina vs gliburide. Los estudios que compararon metformina vs insulina, no encontraron diferencias significativas para complicaciones neonatales ni para control glicémico. Los estudios que compararon gliburide vs insulina, no encontraron diferencias significativas respecto al control glicémico. Finalmente, los estudios que compararon metformina vs gliburide, no demostraron diferencias significativas en el control glicémico entre ambas drogas.

Con respeto a los posibles efectos adversos fetales de los HGO, sólo un trabajo de los revisados 
(18) evidencia un aumento en la tasa de hipoglicemia neonatal con el uso de gliburide. Este estudio es de diseño deficiente y no cuenta con el poder suficiente para demostrar diferencias en la incidencia de hipoglicemia neonatal, por lo que sus resultados se pueden deber al azar. Los demás trabajos no muestran efectos adversos perinatales.

Nuestro metaanálisis no evidencia diferencias entre HGO e insulina con respecto a la hipoglicemia neonatal. Creemos que los HGO son seguros durante el embarazo, ya que no existe evidencia disponible que demuestre efectos adversos fetales de su uso. Las sulfonilureas de segunda generación (gliburide y glibenclamida) no cruzan la barrera placentaria, asegurando la ausencia de efectos adversos fetales. Para metformina, HGO que si atraviesa la barrera placentaria, existe seguimiento del $\mathrm{RN}$ a 18 meses post parto que no evidencia efectos adversos (14).

Con respecto a la eficacia de los HGO para el control glicémico materno, la gran mayoría de los trabajos revisados en nuestro estudio, que evaluaban este resultado como primario, evidencian que los HGO son igualmente efectivos que insulina para el control glicémico. Los dos trabajos que mostraban diferencias significativas en el control glicémico materno $(5,16)$, específicamente en la glicemia postprandial a las 2 horas, lo hacían a favor de los HGO por sobre la insulina. El metaanálisis realizado en nuestro trabajo, evidencia que el tratamiento de la DMG con HGO presenta glicemias postprandiales a las 2 horas menores que insulina, pero, que insulina presenta glicemias de ayuno menores que los HGO. Los datos presentados permiten concluir que los HGO son una opción igualmente efectiva que insulina para el control glicémico materno en pacientes con DMG.

Con respecto a la tasa de fallo de los $\mathrm{HGO}$ en el tratamiento de la DMG, vemos que esta varía entre un $21 \%$ y $46,3 \%$ para el uso de metformina, y entre un $4 \%$ y $29 \%$ para gliburide. En teoría no existiría una tasa de fallo de control glicémico en pacientes con DMG controladas con insulina, pues este es el estándar de manejo. Sin embargo, en nuestra práctica médica diaria vemos que la insulina también fracasa en el control glicémico de las pacientes con DMG. El aumento indiscriminado de la dosis de insulina para lograr control glicémico llega a un punto en donde el riesgo de hipoglicemia materna severa supera los beneficios del control glicémico adecuado. De utilizar los HGO como primera línea de manejo médico para las pacientes con DMG en que la dieta y ejercicio fracasan en controlar la glicemia, evitaríamos el uso de insulina en estas pacientes entre un $53,7 \%$ a $96 \%$ de los casos. Los datos pre- sentados apoyan el uso de HGO para el manejo de la DMG previo a la utilización de insulina, ya que su uso sería seguro durante el embarazo, logran el control glicémico de forma igualmente efectiva que la insulina y se evitaría el uso de insulina en al menos la mitad de las pacientes con DMG que fracasan en el manejo con dieta y ejercicio.

Con respecto a qué HGO utilizar, creemos que la opción a seguir es la utilización de sulfonilureas de segunda generación (gliburide o glibenclamida), ya que logran control glicémico en forma igualmente efectiva a la insulina, no cruza la barrera placentaria y fracasan significativamente menos en el tratamiento de la DMG que la metformina $(27,6 \%$ vs $38,5 \%, p<0,0001 ;$ IC95\% 1,21-1,60).

\section{CONCLUSIÓN}

Los HGO son un tratamiento seguro y efectivo para la DMG. Recomendamos el uso de sulfonilureas de segunda generación (gliburide o glibenclamida) para el tratamiento de las pacientes con DMG que fracasan en su control glicémico con dieta y ejercicio, evitando así el uso de insulina en hasta un $71 \%$ de las pacientes con DMG.

\section{REFERENCIAS}

1. Committee opinion No. 504. Screening and diagnosis of gestational diabetes mellitus. Obstet Gynecol 2011;118(3):751-3.

2. Dhulkotia JS, Ola B, Fraser R, Farrell T. Oral hypoglycemic agents vs insulin in management of gestational diabetes: a systematic review and metaanalysis. Am J Obstet Gynecol 2010;203(5):457.e1-457.e9.

3. Langer O, Yogev Y, Most O, Xenakis EMJ. Gestational diabetes: The consequences of not treating. Am J Obstet Gynecol 2005;192(4):989-97.

4. Crowther CA, Hiller JE, Moss JR, McPhee AJ, Jeffries WS, Robinson JS, et al. Effect of treatment of gestational diabetes mellitus on pregnancy outcomes. N Engl J Med 2005;352(24):2477-86.

5. Rowan JA, Hague WM, Gao W, Battin MR, Moore MP, MiG Trial Investigators. Metformin versus insulin for the treatment of gestational diabetes. $\mathrm{N}$ Engl $\mathrm{J}$ Med 2008;358(19):2003-15.

6. Silva JC, Pacheco C, Bizato J, de Souza BV, Ribeiro TE, Bertini AM. Metformin compared with glyburide for the management of gestational diabetes. Int J Gynaecol Obstet 2010;111(1):37-40.

7. Nicholson W, Bolen S, Witkop CT, Neale D, Wilson L, Bass E. Benefits and risks of oral diabetes agents compared with insulin in women with gestational diabetes: a systematic review. Obstet Gynecol 2009;113(1):193-205.

8. Moretti ME, Rezvani M, Koren G. Safety of glyburide for gestational diabetes: a meta-analysis of pregnancy outcomes. Ann Pharmacother 2008;42(4):483-90. 
9. Langer O, Conway DL, Berkus MD, Xenakis EM, Gonzales $\mathrm{O}$. A comparison of glyburide and insulin in women with gestational diabetes mellitus. $\mathrm{N}$ Engl $\mathrm{J}$ Med 2000;343(16):1134-8.

10. Silva JC, Fachin DRRN, Coral ML, Bertini AM. Perinatal impact of the use of metformin and glyburide for the treatment of gestational diabetes mellitus. J Perinat Med 2012;40(3):225-8.

11. ljäs $H$, Vääräsmäki M, Morin-Papunen L, Keravuo R, Ebeling T, Saarela T, et al. Metformin should be considered in the treatment of gestational diabetes: a prospective randomised study. BJOG 2010;118(7):880-5.

12. Moore LE, Clokey D, Rappaport VJ, Curet LB. Metformin compared with glyburide in gestational diabetes: a randomized controlled trial. Obstet Gynecol 2010;115(1):55-9.

13. Glueck CJ, Wang P, Goldenberg N, Sieve-Smith L. Pregnancy outcomes among women with polycystic ovary syndrome treated with metformin. Hum Reprod 2002;17(11):2858-64.
14. Glueck CJ. Height, weight, and motor-social development during the first 18 months of life in 126 infants born to 109 mothers with polycystic ovary syndrome who conceived on and continued metformin through pregnancy. Hum Reprod 2004;19(6):1323-30.

15. Moore LE, Briery CM, Clokey D, Martin RW, Williford NJ, Bofill JA, et al. Metformin and insulin in the management of gestational diabetes mellitus: preliminary results of a comparison. J Reprod Med 2007;52(11):1011-5.

16. Ogunyemi D, Jesse M, Davidson M. Comparison of glyburide versus insulin in management of gestational diabetes mellitus. Endocr Pract 2007;13(4):427-8.

17. Anjalakshi C, Balaji V, Balaji MS, Seshiah V. A prospective study comparing insulin and glibenclamide in gestational diabetes mellitus in Asian Indian women. Diabetes Res Clin Pract 2007;76(3):474-5.

18. Bertini AM, Silva JC, Taborda W, Becker F, LemosBebber FR, ZuccoViesi JM, et al. Perinatal outcomes and the use of oral hypoglycemic agents. J Perinat Med 2005;33(6):519-23. 